
\title{
3 Research S Suare \\ Precision and Agreement of Central Corneal \\ Thickness Measured with E-pach, A-scan, Corvis ST and Pentacam
}

\section{Anqi Liu}

General Hospital of People's Liberation Army

\section{Zequan Xu}

Military General Hospital of Beijing PLA

Jingjing Wang

Military General Hospital of Beijing PLA

Feng Liu

Military General Hospital of Beijing PLA

\section{Yanming Jiang}

rocket force general hospital of chinese people liberation army

\section{Min Yang}

Chinese People's Liberation Army 81 group hospital

\section{Lina Mei}

Military General Hospital of Beijing PLA

\section{Mei Ge}

Military General Hospital of Beijing PLA

\section{Qinghua Yang}

Military General Hospital of Beijing PLA

\section{Yifei Huang}

Military General Hospital of Beijing PLA

Liqiang Wang ( $\square$ liqiangw301@163.com )

Chinses People's Liberation Army General Hospital

\section{Research article}

Keywords: E-pach; central corneal thickness; pachymetry; repeatability; reproducibility, agreement

Posted Date: March 28th, 2019

DOI: https://doi.org/10.21203/rs.2.508/v1 
License: (c) (i) This work is licensed under a Creative Commons Attribution 4.0 International License. Read Full License 


\section{Abstract}

Purpose To assess the precision (repeatability and reproducibility) and agreement of central corneal thickness (CCT) measurements by E-pach, Pentacam, Corvis ST and A-scan devices in healthy eyes. Methods Instrument diagnostic test study. A total of 100 patients were enrolled to measure right-eye CCT by E-pach, Pentacam, Corvis ST, and A-scan devices. To assess repeatability and reproducibility, the testretest repeatability (TRT) and intraclass correlation coefficient (ICC) were calculated. The agreement among the four devices was evaluated with Bland-Altman plots. Results The E-pach showed repeatability (ICC=0.9981), interobserver reproducibility (ICC=0.9971) and intersession reproducibility (ICC=0.9825); the Pentacam, Corvis ST and A-scan also showed similar repeatability (all ICC $\geq 0.9679$ ), interobserver reproducibility (all ICC $\geq 0.9730$ ) and intersession reproducibility (all ICC $\geq 0.9647$ ). However, the E-pach yielded CCT values that were obviously lower than those of the A-scan and Pentacam $(P<0.001)$ but higher than those of the Corvis ST $(P<0.001)$. The 95\% limits of agreement (LoA) in the Bland-Altman plots were $44.5 \mu \mathrm{m}$ (E-pach vs. A-scan), $34.9 \mu \mathrm{m}$ (E-pach vs. Corvis CT) and $32.5 \mu \mathrm{m}$ (E-pach vs. Pentacam). Conclusions The E-pach is a portable, reliable and inexpensive pachymeter. However, the CCT values obtained from the E-pach are not interchangeable with those from Pentacam, Corvis ST and Ascan devices.

\section{Introduction}

The measurement of central corneal thickness (CCT) is a critical in many steps for refractive surgery such as preoperative and postoperative evaluation, as well as intraoperative evaluation. [1, 2] It is worth mentioning that measuring CTT in intraoperative assessment is important to improve the accuracy of refractive surgery and reduce complications, which is sometimes ignored by some ophthalmologists. Furthermore, CCT measurement is an indispensable test for the diagnosis of glaucoma.[3] In addition, CCT plays an important role in the treatment and evaluation of endothelial diseases, such as corneal endothelial dystrophy and corneal failure after corneal transplantation.[4] At present, various methods are used for the CCT measurement, such as ultrasound pachymetry (USP), Scheimflug photography, optical coherence tomography and so on. [5-9] Most often, USP is regarded as the gold-standard technique for measuring CCT. [10-12]

Many studies have evaluated the precision (repeatability and reproducibility) and agreement of CCT measurements among the abovementioned devices. [7, 8, 11-26] The results, however, have not always been consistent.

The E-pach ${ }^{\mathrm{TM}}$ (Sonogage Inc.) is a new, portable ultrasonic pachymeter. To the best of our knowledge, no study has evaluated the precision of this instrument or comprehensively compared it to other similar instruments. In the present study, we estimated the precision (repeatability and reproducibility) of CCT measurement by E-pach and compare its agreement with Pentacam, Corvis ST, and A-scan devices.

\section{Subjects And Methods}




\section{Subjects}

This study was approved by the Office of Research Ethics at the General Hospital of People liberation Army (PLA), and informed consent was acquired from all subjects. We enrolled healthy subjects recruited from a group of volunteers at the General Hospital of PLA from June 6th to July 6th.

A total of 100 healthy subjects participated in the study and met the following criteria: (1) good fixation ability of the right eye; (2) good communication. The exclusion criteria were: (1) corneal abnormal topographic map; (2) any history of eye surgery or trauma; (3) active eye disease; (4) systemic diseases with eye symptoms; (5) intraocular pressure (IOP) >21 mmHg; (6) recent contact lens wear (rigid contact lens within four weeks and soft contact lens within two weeks.

\section{Measurement protocol}

The measurement of precision and agreement strictly followed the British Standards Institute and the International Organization for Standardization (BSISO).[27] All subjects underwent a complete ophthalmologic examination, including refraction, uncorrected and best corrected distance vision (VA), slit-lamp microscopy, and fundus examinations).

Besides, CCT was measured by E- pach, Pentacam, Corvis ST, and A-scan. To avoid any effect of the ultrasound probe and topical anaesthetic on the cornea, the two noncontact pieces of equipment (Pentacam and Corvis ST) were used first.

All measurements were performed from 8:00 to 10:00 or from 14:00 to 16:00. In the first session, each subject had three consecutive measurements conducted by observer $A$ and observer $B$ to assess repeatability and interobserver reproducibility. In the second session (one week later), each subject had an additional three consecutive scans by one observer $A$ to assess intersession reproducibility. .

\section{Instruments}

The E-pach ${ }^{\mathrm{TM}}$ (Sonogage, Inc.) is a new, hand-held ultrasonic pachymeter. Its transducer, of greater than 50 $\mathrm{MHz}$, results in 2.5 times the accuracy of $20 \mathrm{MHz}$ pachymeter transducers.

The A-scan ultrasound pachymeter (Pachette 500, DGH Technology Inc, Frazer, PA, USA) was used to evaluate CCT values.

The Pentacam $₫$ Oculus Inc. $₫$ Germany $₫$ Standard uses a rotating Scheimpflug camera (180 degrees) to

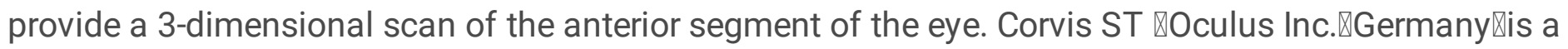
non-contact tonometer equipped with an optical pachymetry function.

\section{Data and Statistical Analysis}

All statistics were calculated using SPSS software for Windows version 21 (SPSS Inc., Chicago, IL, U.S.) and MedCalc Statistical Software version 11.0 (MedCalc Software, Inc., Mariakerke, Belgium). We 
calculated the average of each common parameter $( \pm S D)$ from the four devices.

We calculated the within-subject standard deviation (Sw), test-retest repeatability (TRT), and intraclass correlation coefficient (ICC) to assess precision (including repeatability, interobserver reproducibility and intersession reproducibility). The TRT, which defined as $2.77 \mathrm{Sw}$, represents the $95 \%$ confidence interval $(\mathrm{Cl})$ around the Sw within which $95 \%$ of measurements should occur.

The ICC is a reliability coefficient that evaluates the consistency of datasets of repeated measurements (ICC < 0.75: low; 0.75 $\leq$ ICC $\leq$ 0.90: moderate; and ICC > 0.9: good). [28, 29] We carried out Bland-Altman plots to assess the agreement of the four devices. Two instruments may be considered interchangeable if the $95 \%$ limits of agreement (LoA) is not clinically significant $(10 \mu \mathrm{m})$, which were defined as \pm 1.96 standard deviation.

\section{Results}

In this study, 100 healthy subjects (42 males) were enrolled. The mean age was $28.78 \pm 4.01$ years (range, 18-54).

\section{Repeatability of Corneal Thickness Measurements}

Table 1 displays the mean values, repeatability (Sw), TRT (2.77Sw) and ICCs of three consecutive corneal thickness measurements made by the observer A and obtained by E-pach, A-scan, Corvis CT and Pentacam devices. All ICCs were more than 0.96 .

\section{Interobserver Reproducibility of Corneal Thickness Measurements}

Table 2 displays the mean values, repeatability (Sw), TRT $(2.77 \mathrm{Sw}$ ) and ICCs of the corneal thickness measurements between the two observers obtained by the four instruments. All ICCs were more than 0.97 .

\section{Intrasession Reproducibility of Corneal Thickness Measurements}

Table 3 displays the mean values, repeatability (Sw), TRT (2.77Sw) and ICCs for corneal thickness measurements between the two sessions (only by observer $A$ ) obtained by the four instruments. All ICCs were more than 0.96 .

\section{Comparison of Corneal Thickness Measurements Obtained by E-pach, A-scan, Corvis ST and Pentacam}

Table 4 displays the differences in corneal thickness measurements obtained from the four devices.

Corneal thickness values obtained with the E-pach were statistically smaller than those obtained with the A-scan $(P<0.001)$. Meanwhile, the 95\% LoA in the Bland-Altman plots was $44.5 \mu \mathrm{m}$ (Figure 1A). 
Corneal thickness values obtained with the E-pach were statistically larger than those obtained with the Corvis CT ( $<$ < 0.001). Meanwhile, the 95\% LoA in the Bland-Altman plots was $34.9 \mu \mathrm{m}$ (Figure 1B).

Corneal thickness values obtained with the E-pach were statistically smaller than those obtained with the Pentacam $(P<0.001)$. Meanwhile, the 95\% LoA in the Bland-Altman plots was $32.5 \mu \mathrm{m}$ (Figure 1C).

Corneal thickness values obtained with the A-scan were statistically larger than those obtained with the Corvis CT $(P<0.001)$. Meanwhile, the 95\% LoA in the Bland-Altman plots was $52.8 \mu \mathrm{m}$ (Figure 1D).

Corneal thickness values obtained with the A-scan were statistically larger than those obtained with the Pentacam $(P<0.001)$. Meanwhile, the 95\% LoA in the Bland-Altman plots was $50.1 \mu \mathrm{m}$ (Figure 1E).

Corneal thickness values obtained with the Corvis CT were statistically larger than those obtained with the Pentacam $(P<0.001)$. Meanwhile, the $95 \%$ LoA in the Bland-Altman plots was $40.7 \mu \mathrm{m}$ (Figure $1 \mathrm{~F})$.

\section{Discussion}

As mentioned above, accurate CCT measurements are essential for refractive surgery and the diagnosis and management of patients with glaucoma and endothelial disorders. Although the E-pach is a new, portable pachymeter, no study has yet to investigate the precision and agreement of this instrument. Our results demonstrated that the E-pach had great repeatability and reproducibility in CCT measurements. The agreement between the E-pach and other instruments is, however, not good. The E-pach yielded CCT values that were significantly lower than those from the A-scan and Pentacam but significantly higher than those from the Corvis ST.

Our study showed great repeatability by the E-pach: the TRT value was $3.7007 \mu \mathrm{m}$, and the ICC value was 0.9981 (far beyond 0.9). Repeatability of CCT values measured by the A-scan, Corvis ST and Pentacam devices were almost equally great.

The repeatability of CCT values measured by the A-scan, Corvis ST and Pentacam devices in the present study are consistent with previous studies. Our study showed satisfying repeatability using the A-scan: the TRT value was $14.0345 \mu \mathrm{m}$, and the ICC value was 0.9797 . Bao et al.[30] assessed the repeatability of CCT with the A-scan. They found that the TRT value was $7.65 \mu \mathrm{m}$, and the ICC value was 0.994 . Moreover, Gokcinar et al.[31] also demonstrated that the ICC value of the A-scan is 0.994 . Our study also showed satisfying repeatability by Corvis ST: the TRT value was $15.0946 \mu \mathrm{m}$, and the ICC value was 0.9679 . Yu et al.[32] and Chen et al.[33] both assessed the repeatability of CCT with the Corvis ST, they found that the TRT values were $13.0 \mu \mathrm{m}$ and $12.56 \mu \mathrm{m}$, and the ICC values were 0.971 and 0.99 . Meanwhile, our study also showed satisfying repeatability using the Pentacam: the TRT value was $11.3098 \mu \mathrm{m}$, and the ICC value was 0.9852. Huang et al.[17], Wiswanathan et al.[18], and Crawford et al.[19] evaluated the repeatability of CCT with Pentacam and indicated that the ICC values were $0.980,0.984$ and 0.979 , respectively. 
In addition to repeatability, interobserver and intersession reproducibility of CCT measurements acquired with the abovementioned devices were also estimated. For interobserver reproducibility of the E-pach, the TRT value was $5.0082 \mu \mathrm{m}$, and the ICC value was 0.9971 ; for intersession reproducibility of the E-pach, the TRT value was $12.5110 \mu \mathrm{m}$, and the ICC value was 0.9825 . As expected, the intersession reproducibility was slightly worse than the interobserver reproducibility; however, the general reproducibility was far beyond good (ICC $=0.90)$. Reproducibility of CCT values measured by the A-scan, Corvis ST and Pentacam devices were almost equally great.

Reproducibility of CCT values measured by the A-scan, Corvis ST and Pentacam devices in the present study were similar to previous studies. Nam et al.[34] evaluated the interobserver reproducibility of CCT measurements using A-scan. They found a TRT value of $5.2 \mu \mathrm{m}$ and an ICC value of 0.995. Ali et al.[35] evaluated the intersession reproducibility of CCT measurement using Corvis ST. They found a TRT value of $11 \mu \mathrm{m}$ and an ICC value of 0.980. Bourges et al.[22] assessed the interobserver and intersession reproducibility using Pentacam at ICC values of 0.993 and 0.980 , respectively.

When the CCT readings were compared among the four devices, a significant difference in average CCT values was observed. Specifically, in the results of the T test, average CCT values of the A-scan were significantly higher than those of the Pentacam; however, these Pentacam values were significantly higher than those of the E-pach, which were significantly higher than those of the Corvis CT. The results of the Bland-Altman plots were consistent with the T test.

Several previous studies have reported that CCT readings of the A-scan are higher than those of the Pentacam and Corvis ST devices. Smedowski et al.[36] assessed the agreement of CCT readings using the Pentacam, Corvis ST and A-scan devices. They indicated that the mean CCT value was the highest for the A-scan and the lowest for the Corvis ST. Another two studies $[13,24]$ also reported that a small underestimation of CCT values with the Pentacam compared to the A-scan. There are two possible explanations for this underestimation: (1) the CCT readings by the A-scan were affected after giving topical $0.5 \%$ proparacaine hydrochloride, which increased corneal thickness $(8.6 \mu \mathrm{m}$ increase in 80 seconds) [37]; (2) the accuracy of the A-scan is influenced by whether the probe is placed as perpendicular as possible to the centre of the cornea.[38-40] The results, however, are still controversial. For example, Tai et al.[41] showed that the CCT value of the Pentacam is overestimated by $10 \mu \mathrm{m}$ compared with that of the A-scan.

Despite controversy, we did obtain some valid results. Because the CCT readings of the E-pach were statistically and clinically different $(>10 \mu \mathrm{m})$ from those of the other instruments (whether by $T$ test or by Bland-Altman), the E-pach is not interchangeable with them.

There were some limitations in our present study. We only evaluated the precision and agreement of CCT measurements in normal eyes. In further research, ocular disorders including glaucoma, myopia, keratoconus, leucoma, or post-refractive surgery could be included. In addition, other types of instruments could be included in future studies. 
In conclusion, the new versions of the A-scan and E-pach are portable, relatively inexpensive and reliable (our study indicated that the E-pach displayed great repeatability and reproducibility). However, the CCT values obtained from the E-pach are not interchangeable with those from the traditional A-scan, Pentacam and Corvis ST devices.

\section{Declarations}

\section{Acknowledgements}

This research was supported Supported by the Chinese Capital Clinical Features Key Project - Clinical Application on Chinese Keratoprosthesis (Project No: Z161100000516012), National Natural Science Foundation of China (Grant No. 81770887), and National Natural Science Foundation of China (Grant No. 81670830).

\section{Availability of data and material:}

The datasets used and/or analysed during the current study available from the corresponding author on reasonable request.

\section{Author Contributions:}

Conceived and designed the experiments: Zequan Xu, Liqiang Wang. Performed the experiments: Anqi liu, Jingjing wang, Feng Liu. Analysed the data: Zequan Xu. Contributed reagents/materials/analysis tools: Yanming Jiang, Min yang, Lina Mei, Mei Ge, Qinghua Yang. Wrote the paper: Anqi Liu Zequan Xu. Critical revision of the manuscript: Liqiang Wang, Yifei Huang.

\section{Ethics approval and consent to participate}

The Declaration of Helsinki was strictly followed in all procedures. Written informed consent was obtained from all subjects. And the study was approved by the Medical Ethics Committee of the General Hospital of People liberation Army (PLA).

\section{Consent to Publish}

Not applicable

\section{Conflict of interest statement}

All the authors have no conflicts of interest.

\section{References}

1. Randleman JB, Woodward M, Lynn MJ, Stulting RD: Risk assessment for ectasia after corneal refractive surgery. Ophthalmology 2008, 115(1):37-50. 
2. DL K, MO P: Central corneal pachymetry in patients undergoing laser in situ keratomileusis. Ophthalmology 1999, 106(11):2216-2220.

3. Kaushik S, Pandav SS, Banger A, Aggarwal K, Gupta A: Relationship between corneal biomechanical properties, central corneal thickness, and intraocular pressure across the spectrum of glaucoma. Am J Ophthalmol 2012, 153(5):840-849 e842.

4. Mohamed Abou Shousha SHY, and Mohamed S. Sayed: In Vivo Characteristics of Corneal Endothelium/Descemet' s membrane Complex for the Diagnosis of Corneal Graft Rejection. Am J Ophthalmol2017, 178:27-37.

5. Marcus Ang MB, René M. Werkmeister, et al.: Anterior Segment Optical Coherence Tomography. Progress in Retinal and Eye Research 2018, 66:132-156.

6. LOREN S. SEERY CBN, JAY W. MCLAREN, et al.: Graft Thickness, Graft Folds, and Aberrations After Descemet Stripping Endothelial Keratoplasty for Fuchs Dystrophy. Am J Ophthalmol 2011:152:910-916.

7. Meyer JJ, Gokul A, Vellara HR, Prime Z, McGhee CN: Repeatability and Agreement of Orbscan II, Pentacam HR, and Galilei Tomography Systems in Corneas With Keratoconus. Am J Ophthalmo/2017, 175:122-128.

8. Xu Z, Peng M, Jiang J, Yang C, Zhu W, Lu F, Shen M: Reliability of Pentacam HR Thickness Maps of the Entire Cornea in Normal, Post-Laser In Situ Keratomileusis, and Keratoconus Eyes. Am J Ophthalmol 2016, 162:74-82 e71.

9. TUKEZBAN HUSEYNOVA GOW, CYNTHIA ROBERTS, et al.: Corneal Biomechanics as a Function of Intraocular Pressure and Pachymetry by Dynamic Infrared Signal and Scheimpflug Imaging Analysis in Normal Eyes. Am J Ophthalmol 2014:157:885-893.

10. Huseynova T, Waring GOt, Roberts C, Krueger RR, Tomita M: Corneal biomechanics as a function of intraocular pressure and pachymetry by dynamic infrared signal and Scheimpflug imaging analysis in normal eyes. Am J Ophthalmol 2014, 157(4):885-893.

11. S M, CK L, SK R, AC C, CY C, DS L: Agreement among 3 methods to measure corneal thickness: ultrasound pachymetry, Orbscan II, and Visante anterior segment optical coherence tomography.\%A Li EY. Ophthalmology 2007, 114(10):1842-1847.

12. $\mathrm{P} \mathrm{A}, \mathrm{K}$ W: Comparison of central corneal thickness measurements by ultrasound pachymetry and 2 new devices, Tonoref III and RS-3000. International ophthalmology 2018, undefined(undefined): undefined.

13. Clare O'Donnell aCM-C: Agreement and Repeatability of Central Thickness Measurement in Normal Corneas Using Ultrasound Pachymetry and the OCULUS Pentacam. Cornea 2005:24:920-924. 
14. Ciolino JB, Khachikian SS, Belin MW: Comparison of comeal thickness measurements by ultrasound and scheimpflug photography in eyes that have undergone laser in situ keratomileusis. Am J Ophthalmol 2008, 145(1):75-80.

15. C M-C: Agreement and repeatability of central thickness measurement in normal corneas using ultrasound pachymetry and the OCULUS Pentacam.\%A O'Donnell C. Cornea 2005, 24(8):920-924.

16. KW K, CM N, V S: Central corneal thickness measurements with different imaging devices and ultrasound pachymetry.\%A Tai LY. Cornea 2013, 32(6):766-771.

17. Huang J, Ding X, Savini G, Pan C, Feng Y, Cheng D, Hua Y, Hu X, Wang Q: A Comparison between Scheimpflug imaging and optical coherence tomography in measuring corneal thickness. Ophthalmology 2013, 120(10):1951-1958.

18. Viswanathan D, Kumar NL, Males JJ, Graham SL: Comparative analysis of corneal measurements obtained from a Scheimpflug camera and an integrated Placido-optical coherence tomography device in normal and keratoconic eyes. Acta ophthalmologica 2015, 93(6):e488-494.

19. Crawford AZ, Patel DV, McGhee CN: Comparison and repeatability of keratometric and corneal power measurements obtained by Orbscan II, Pentacam, and Galilei corneal tomography systems. Am J Ophthalmol 2013, 156(1):53-60.

20. Q W, S C, G S, W L, Y F, Y Y, J H: Comparison and evaluation of central corneal thickness using 2 new noncontact specular microscopes and conventional pachymetry devices.\%A Bao F. Cornea 2014, 33(6):576-581.

21. E Y, N O, S Y, Z O: Agreement and repeatability of central corneal thickness measurements by four different optical devices and an ultrasound pachymeter.\%A Gokcinar NB. International ophthalmology 2018, undefined(undefined): undefined.

22. Bourges JL, Alfonsi N, Laliberte JF, Chagnon M, Renard G, Legeais JM, Brunette I: Average 3dimensional models for the comparison of Orbscan II and Pentacam pachymetry maps in normal corneas. Ophthalmology 2009, 116(11):2064-2071.

23. CY I, HK L, EK K, TI K, KY S: Accuracy of RTVue optical coherence tomography, Pentacam, and ultrasonic pachymetry for the measurement of central corneal thickness.\%A Nam SM. Ophthalmology 2010, 117(11):2096-2103.

24. Gonzalez-Perez J, Gonzalez-Meijome JM, Rodriguez Ares MT, Parafita MA: Central corneal thickness measured with three optical devices and ultrasound pachometry. Eye \& contact lens 2011, 37(2):66-70.

25. B W, D T, K K, E W: Comparison of three intraocular pressure measurement methods including biomechanical properties of the cornea.\%A Smedowski A. Investigative ophthalmology \& visual science 2014, 55(2):666-673. 
26. E A, M G, G M, S G, N O: Intraobserver and interobserver reproducibility in the evaluation of ultrasonic pachymetry measurements of central corneal thickness.\%A Miglior S. The British journal of ophthalmology 2004, 88(2):174-177.

27. Bland JM, Altman D: Statistical methods for assessing agreement between two methods of clinical measurement. The lancet 1986, 327(8476):307-310.

28. McAlinden C, Khadka J, Pesudovs K: Statistical methods for conducting agreement (comparison of clinical tests) and precision (repeatability or reproducibility) studies in optometry and ophthalmology. Ophthalmic and Physiological Optics 2011, 31(4):330-338.

29. Y H, W Q, G L, Q W: Precision and agreement of higher order aberrations measured with ray tracing and Hartmann-Shack aberrometers.\%A Xu Z. BMC ophthalmology 2018, 18(1):18.

30. von Sonnleithner C, Bergholz R, Gonnermann J, Klamann MK, Torun N, Bertelmann E: Clinical results and higher-order aberrations after 1.4-mm biaxial cataract surgery and implantation of a new aspheric intraocular lens. Ophthalmic research 2015, 53(1):8-14.

31. Gokcinar NB, Yumusak E, Ornek N, Yorubulut S, Onaran Z: Agreement and repeatability of central corneal thickness measurements by four different optical devices and an ultrasound pachymeter. International ophthalmology 2018.

32. Yu A, Zhao W, Savini G, Huang Z, Bao F, Lu W, Wang Q, Huang J: Evaluation of Central Corneal Thickness Using Corneal Dynamic Scheimpflug Analyzer Corvis ST and Comparison with Pentacam Rotating Scheimpflug System and Ultrasound Pachymetry in Normal Eyes. Journal of ophthalmology 2015, 2015:767012.

33. Chen X, Stojanovic A, Hua Y, Eidet JR, Hu D, Wang J, Utheim TP: Reliability of corneal dynamic scheimpflug analyser measurements in virgin and post-PRK eyes. PloS one 2014, 9(10):e109577.

34. Nam SM, Im CY, Lee HK, Kim EK, Kim TI, Seo KY: Accuracy of RTVue optical coherence tomography, Pentacam, and ultrasonic pachymetry for the measurement of central corneal thickness. Ophthalmology 2010, 117(11):2096-2103.

35. Ali NQ, Patel DV, McGhee CN: Biomechanical responses of healthy and keratoconic corneas measured using a noncontact scheimpflug-based tonometer. Investigative ophthalmology \& visual science 2014, 55(6):3651-3659.

36. Adrian Smedowski BW, Dorota Tarnawska, et al.: Comparison of Three Intraocular Pressure Measurement Methods Including Biomechanical Properties of the Cornea. Invest Ophthalmol Vis Sci 2014:55:666-673.

37. Sang Min Nam HKL, Eung Kweon Kim, and Kyoung Yul Seo: Comparison of Corneal Thickness After the Instillation of Topical Anesthetics: Proparacaine Versus Oxybuprocaine. Cornea 2006:25:51-54. 
38. Nader Nassiri KS, Sare Safi, et al.: Central Corneal Thickness in Highly Myopic Eyes: Inter-device Agreement of Ultrasonic Pachymetry, Pentacam and Orbscan II Before and After Photorefractive Keratectomy. J Ophthalmic Vis Res 2014:9 (1): 14-21.

39. S Miglior EA, M Guareschi, et al.: Intraobserver and interobserver reproducibility in the evaluation of ultrasonic pachymetry measurements of central corneal thickness. Br J Ophthalmol 2004:88:174-177.

40. Matthew M. Marsich aMAB: The Repeatability of Corneal Thickness Measures. Cornea 2000:19(16): $792-795$.

41. Lai-Yong Tai K-WK, Choung-Min Ng, et al: Central Corneal Thickness Measurements With Different Imaging Devices and Ultrasound Pachymetry. Cornea 2013:32:766-771.

\section{Tables}

Table 1. The intraobserver repeatability of CCT measurements by different devices

\begin{tabular}{lllll} 
Instrument & Mean \pm SD & Sw (D) & 2.77 Sw (D) & ICC \\
\hline E-pach & $545.0000 \pm 33.5456$ & 1.3360 & 3.7007 & $\mathbf{0 . 9 9 8 1}(\mathbf{0 . 9 9 7 3 - 0 . 9 9 8 6 )}$ \\
\hline A-scan & $567.50000 \pm 34.8681$ & 5.0666 & 14.0345 & $\mathbf{0 . 9 7 9 7 ( 0 . 9 7 1 8 - 0 . 9 8 5 7 )}$ \\
\hline Corvis ST & $534.5000 \pm 34.0839$ & 5.4493 & 15.0946 & $\mathbf{0 . 9 6 7 9}(\mathbf{0 . 9 6 7 9 - 0 . 9 8 3 7 )}$ \\
\hline Pentacam & $552.5000 \pm 32.3173$ & 4.1122 & 11.3908 & $\mathbf{0 . 9 8 5 2}(\mathbf{0 . 9 7 9 5 - 0 . 9 8 9 6 )}$
\end{tabular}

Note: SD: standard deviation, Sw: within-subject standard deviation, 2.77Sw: test-retest repeatability (TRT), ICC: intraclass correlation coefficient.

Table 2. The intraobserver reproducibility of CCT measurements by different devices

\begin{tabular}{lllll} 
Instrument & Mean \pm SD & Sw (D) & $2.77 S w(D)$ & ICC \\
\hline E-pach & $536.7067 \pm 33.7245$ & 1.8080 & 5.0082 & $\mathbf{0 . 9 9 7 1}(\mathbf{0 . 9 9 5 7 - 0 . 9 9 8 0 )}$ \\
\hline A-scan & $560.6933 \pm 33.8088$ & 3.7720 & 10.4484 & $\mathbf{0 . 9 8 7 9}(\mathbf{0 . 9 8 2 0 - 0 . 9 9 1 8 )}$ \\
\hline Corvis ST & $530.8417 \pm 34.8129$ & 5.6367 & 15.6137 & $\mathbf{0 . 9 7 3 0 ( 0 . 9 6 0 1 - 0 . 9 8 1 7 )}$ \\
\hline Pentacam & $546.6967 \pm 32.0052$ & 3.5223 & 9.7568 & $\mathbf{0 . 9 8 8 2}(\mathbf{0 . 9 8 2 5 - 0 . 9 9 2 1 )}$
\end{tabular}

Note: SD: standard deviation, Sw: within-subject standard deviation, 2.77Sw: test-retest repeatability (TRT), ICC: intraclass correlation coefficient.

Table 3. The intersession reproducibility of CCT measurements by different devices 


\begin{tabular}{lllll} 
Instrument & Mean \pm SD & Sw (D) & $2.77 S w(D)$ & ICC \\
\hline E-pach & $535.8700 \pm 32.8452$ & 4.5166 & 12.5110 & $\mathbf{0 . 9 8 2 5}(\mathbf{0 . 9 7 4 2 - 0 . 9 8 8 2 )}$ \\
\hline A-scan & $559.7250 \pm 34.4091$ & 5.7353 & 15.8868 & $\mathbf{0 . 9 7 3 3 ( 0 . 9 6 0 6 - 0 . 9 8 2 0 )}$ \\
\hline Corvis ST & $529.9867 \pm 34.3264$ & 6.4782 & 17.9446 & $\mathbf{0 . 9 6 4 7 ( 0 . 9 4 8 2 - 0 . 9 7 6 1 )}$ \\
\hline Pentacam & $545.4884 \pm 31.9253$ & 5.0202 & 13.9060 & $\mathbf{0 . 9 7 6 6}(\mathbf{0 . 9 6 5 4 - 0 . 9 8 4 2 )}$
\end{tabular}

Note: SD: standard deviation, Sw: within-subject standard deviation, 2.77Sw: test-retest repeatability (TRT), ICC: intraclass correlation coefficient.

Table 4. The agreement of CCT measurements by different devices

\begin{tabular}{llll} 
Instrument & Mean Difference \pm SE & $95 \% \mathrm{Cl}$ & P value \\
\hline E-pach vs. A-scan & $-23.797 \pm 1.137$ & -26.052 to -21.541 & $<0.001$ \\
\hline E-pach vs. Corvis CT & $5.997 \pm 0.891$ & 4.230 to 7.764 & $<0.001$ \\
\hline E-pach vs. Pentacam & $-9.577 \pm 0.827$ & -11.218 to -7.935 & $<0.001$ \\
\hline A-scan vs. Corvis CT & $29.793 \pm 1.355$ & 27.105 to 32.481 & $<0.001$ \\
\hline A-scan vs. Pentacam & $14.220 \pm 1.278$ & 11.684 to 16.756 & $<0.001$ \\
\hline Corvis CT vs. Pentacam & $-15.573 \pm 1.040$ & -17.636 to -13.511 & $<0.001$
\end{tabular}

Note: SE: standard error, Cl: Confidence interval

\section{Figures}


A

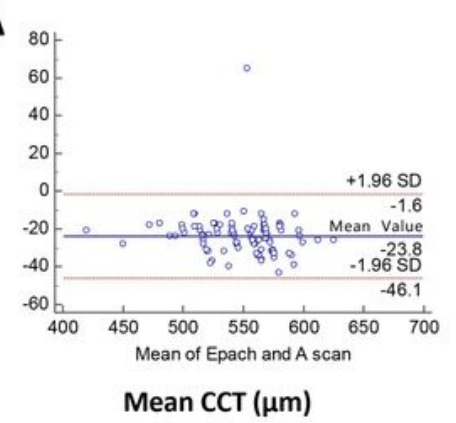

C

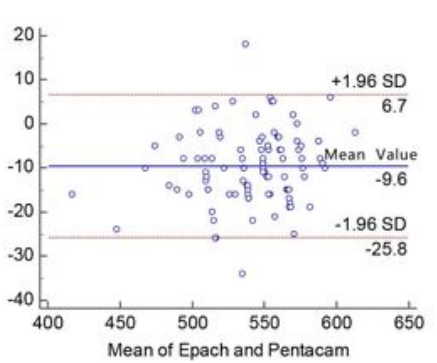

Mean CCT $(\mu \mathrm{m})$

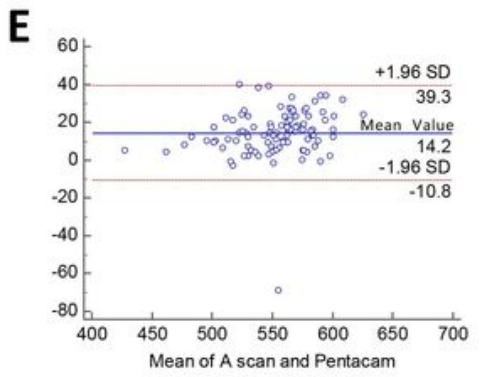

Mean $\mathrm{CCT}(\mu \mathrm{m})$
B

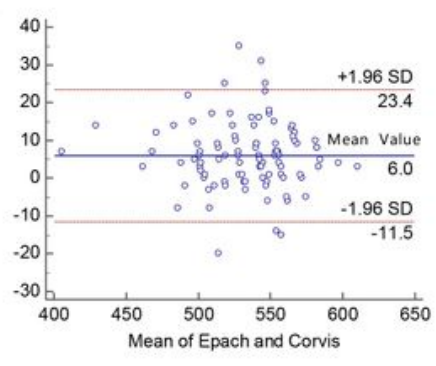

Mean CCT ( $\mu \mathrm{m})$

D

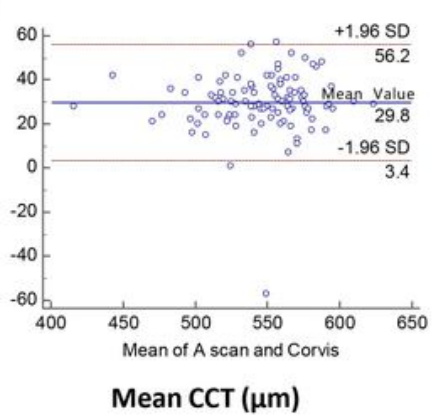

$\mathbf{F}$

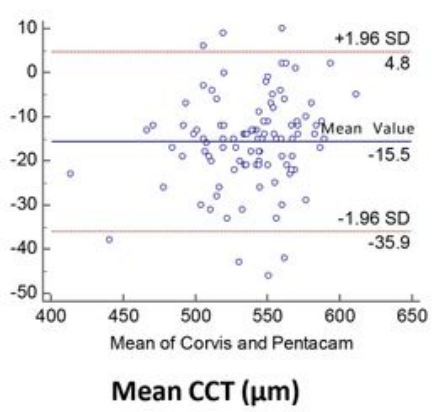

\section{Figure 1}

Bland-Altman plots of the mean central corneal thickness measurement against the differences in a comparison between E-pach vs. A-scan (A), E-pach vs. Corvis ST (B), E-pach vs. Pentacam (C), A-scan vs. Corvis ST (D), A-scan vs. Pentacam (E), Corvis ST vs. Pentacam (F). The solid line indicates the mean difference. The interval between the upper and lower lines represent the 95\% LoA. 